\title{
Translational Science of Psychosis in Parkinson's Disease
}

Syeda Arshiya Farheen ${ }^{1}$, Pooja Shah ${ }^{2}$, Manpreet Gill ${ }^{3}$, Sumayya Ayaz ${ }^{4}$, Satneet Singh ${ }^{5}$, Archana Adikey ${ }^{6}$, Venkatesh Sreeram ${ }^{7}$, Rizwan Ahmed $^{8}$, Mariyah Hussain ${ }^{9}$ and Saeed Ahmed ${ }^{10^{*}}$

${ }^{1}$ Kakatiya Medical college, India;

${ }^{2}$ S.S.R Medical College, Mauritius;

${ }^{3}$ Stanford University School of Medicine, USA:

${ }^{4}$ Dhaka Medical College, Bangladesh;

${ }^{5}$ Tver State Medical Academy, Russia;

${ }^{6}$ King County Hospital Center, USA;

${ }^{7}$ Yale University Diagnostic Radiology, USA;

${ }^{8}$ Liaquat Medical and Dentistry College, Pakistan;

${ }^{9}$ Foundation university Medical College, Pakistan;

${ }^{10}$ Nassau University Medical Center, USA.

\begin{abstract}
Parkinson's disease (PD) is a progressive neurodegenerative disorder characterized by loss of dopamine in the substantia nigra. It is predominantly a motor disorder but has shown to present with psychosis in few cases. Psychotic symptoms result from chronic treatment with few anti- Parkinson's medications, ranging from depression, delusions to visual hallucinations, eventually progressing to auditory hallucinations in a few cases. Presence of psychotic symptoms with loss of insight is indicative of late stage of Parkinson's disease and has poor prognosis. Accurate diagnosis of symptoms can be difficult due to significant overlapping clinical presentations which can be an admixture of underlying disease, exacerbation of pre-existing psychiatric illness or iatrogenic. The article below portrays the complex interplay between Parkinson's disease psychosis, medications and underlying disease process which heralds early identification of the symptoms using diagnostic tools and imaging techniques to improve the health of individuals and the community by "translating" findings into diagnostic tools and medicines. It also highlights the dire need to develop neuroprotective drugs as a mode of primary prevention of psychosis. We cannot underemphasize the need to educate the patient and caregiver to seek medical attention at earliest for prompt identification and application of disease modifying therapies to reduce disability and morbidity associated with it and limit nursing home placements.
\end{abstract}

Keywords: Parkinson's psychosis; Delusions; Nursing home

\section{Introduction}

Psychosis is a significant burden to the individuals and their loved ones worldwide. It is a symptom of various mental illnesses wherein there is an abrupt change in personality with distortion of reality. Discussing the psychosis associated with PD, various factors like pre-existing comorbid conditions, age, and severity of injury, prior subclinical mental health and lack of appropriate support system have exponential influence to the outcome. It starts as a constellation of subtle symptoms which can easily be ignored due to its accordance with general population eventually increasing in magnitude and severity, causing severe distress to the patients and their caregivers. Ambiguity in identifying the warning signs, latency in reaching out to the health care providers and stigma related to it contribute to a very late presentation at the initial visit. This article highlights the delicate balance between management of both psychosis and Parkinson's symptoms, requiring a synergistic approach between Neurologists, Psychiatrists, Psychotherapists and Caregivers. It incorporates biomarkers and imaging techniques which are at the forefront of translational research for Psychosis in PD patients and newer neuroprotective medications that prevent or reduce Psychosis [1,2].

\section{Epidemiology}

Psychotic symptoms in Parkinson's disease are relatively common and generally occur, later in the disease course. However, there is variation in published data regarding the prevalence of psychosis in PD. For example the point prevalence of psychotic symptom in crosssectional studies is $16-23 \%$ in community based studies compared to $21-75 \%$ in clinic based studies [3]. On the other hand, The period prevalence is $17-74 \%$ in long term longitudinal drug trial studies [3]. These high variation in prevalence are mainly due to lack of standardized diagnostic tools, differences in study design, study population, and data collection [4]. Also, there is variation in study population. Most published studies comprised patients from movement disorder clinics with a few studies which included community based samples. Finally, there is little geographical variations with a lack of data from African countries or Asian sub regions [3]. In spite of these limitations several studies have reported psychosis in PD can occur in up to $60 \%$ of patients, regardless of their cognitive status $[3,5,6]$.

\section{Pathophysiology of Psychosis in PD}

The pathophysiology of PD psychosis is still poorly understood. Both extrinsic factor (pharmacological intervention) and intrinsic factor (related to disease itself) may contribute to the development of psychotic symptoms in PD. Traditionally psychosis has been considered as a dopaminergic drug-induced phenomenon, but factors intrinsic to the disease process including Lewy body deposition in the limbic system,

*Corresponding author: Ahmed S, Nassau University Medical Center, USA, Tel: 93298445854; E-mail: ahmedsaeedmd@gmail.com

Received: Septemeber 24, 2015; Accepted: Septemeber 27, 2015; Published: Novomber 15, 2015

Citation: Farheen SA, Shah P, Gill M, Ayaz S, Singh S, et al. (2015) Translational Science of Psychosis in Parkinson's Disease. Clin Depress 1: 102.

Copyright: (c) 2015 Farheen SA, et al. This is an open-access article distributed under the terms of the Creative Commons Attribution License, which permits unrestricted use, distribution, and reproduction in any medium, provided the original author and source are credited. 
cholinergic deficits and impairments of primary visual processing are also contributing factors for visual hallucinations and delusions. The psychotic symptoms of PD are the clinical manifestations of the extensive degeneration of non-dopaminergic systems including several brainstem nuclei (raphe nucleus, locus ceruleus, dorsal vagal nucleus), limbic and neocortical structures, as well as the peripheral autonomic system as evidenced by the post mortem evaluation of PD brains.

\section{The role of dopaminergic medications}

Dopamine has been most consistently recognized as an important neurotransmitter of psychosis. Increased sensitivity of dopamine in mesolimbic and mesocortical areas is involved in the generation of psychosis) and the most commonly explained mechanism of PD psychosis centered on dopaminergic medications used for the motor symptom of PD [2]. The mechanism by which anti-parkinsonism drugs increase susceptibility of psychosis involves hyper sensitization of dopamine receptors in the nigrostriatal pathway leading to dysfunction of limbic structures [7]. In fact, all PD medications including dopaminergic medications, Levodopa, as well as non-dopaminergic medications including anticholinergics, amantadine, and MAO-B inhibitors are associated with the development of psychosis in PD [2].

\section{Other neurochemical abnormalities}

Besides Dopamine, other neurotransmitters like Serotonin and Acetylcholine and their interaction also play role in psychosis. In particular, degeneration of some serotonergic pathways may play an important role. The improvement of psychosis with Ondansetron (a 5-HT3 Antagonist) and atypical antipsychotics provide support to this concept [8]. Acetylcholine is another neurotransmitter with a potential role in PD psychosis. Neuropathological studies have shown significant degeneration of cholinergic neurons in PD [9]. Also, cholinergic deficit in dementia with Lewy Body has been linked to psychosis and the ratio of 5-hydroxyindoleacetic acid to choline acetyltransferase was higher in hallucinating patients than in patients without hallucinations [10].

\section{Visual processing deficits}

Impaired visual processing has been linked to visual hallucinations in Parkinson's disease. Various abnormalities have been discovered in visual networks of PD patients including impaired visual dopaminergic system at the level of retinal dysfunction of visual processing and categorization, deficits in color discrimination and contrast sensitivity [11]. These abnormalities may facilitate the onset of visual hallucination in PD patient.

\section{Sleep disturbances}

Several sleep abnormalities have been found in patients with psychosis in PD including insomnia, daytime sleepiness, vivid dreams. There is a prominent realtionship between reduced REM sleep and psychosis. Polysomnographic studies have shown in a patient who experiences "visual hallucinations has lower sleep efficiency and lower REM sleep time and percentage compare to patient without visual hallucinations [2].

\section{Lewy body deposition}

Several studies have found association between Lewy body deposition and visual hallucination in Parkinson's disease. A recent comparison study found significantly greater Lewy body deposition in the cortical areas and amygdala in patient with visual hallucinations compared to patient without any visual hallucinations [2].

\section{Other factors}

In addition to above, several other factors have been identified as risk factors for the development of psychosis such as cognitive impairment, increased age, disease duration and severity, depression, and autonomic impairment [3].

\section{Clinical Phenomenology of Psychosis}

- It is generally a late manifestation of the disease, but seen bimodal in onset with early onset associated with motor functions and large dose of drugs and late onset seen with cognitive impairment.

- The psychosis is seen with manifestation of mild illusions, vivid dreams, non-disturbing visual hallucinations with clear sensorium in the initial stages.

- The most common psychotic symptom in patients with Parkinson's disease is visual hallucination seen in more than $90 \%$ of affected patients. Initially, the images are of clear sensorium. As disease progresses complex visual hallucinations containing animals, objects or persons are seen and the patient tends to loose insight of his disease and starts acting up on hallucinations. He sees crawling bugs on the patterned carpets or moving insects on the furniture with objects and animals. The hallucinations recur intermittently with frequency of minimum one time in a week with each episode lasting from few seconds to minutes. They generally see in poor lightning conditions with low ambience like "sun downing" or when waking up from sleep.

- The auditory hallucinations occur along with visual hallucinations in small group of patients. They range from small whispers to threatening orders. Unlike, schizophrenia where auditory and visual hallucinations occur independently. The later stage of the disease, is followed by organic psychosis involving olfactory and tactile hallucinations, but always seen along with visual hallucinations $[11,12]$. The patients may have delusions such as paranoid such as spousal infidelity, abandonment, grandiose, religious delusions, and promiscuity. The patients who have insight of the symptoms are termed to have "Benign Hallucinations" and as the disease progresses they eventually lose the insight and develop delusions which is together termed as "Malignant Hallucinations". This is used in measuring the severity of Parkinson's disease in the Unified Parkinson's Disease Rating $[12,13]$.

- The presence of psychosis affects patient's interpersonal relationship and most of the time makes the patient dependent on caregivers and nursing homes.

\section{Dementia}

Dementia is a less common symptom seen in these patients and it takes 10-15 years after the onset of Parkinson's disease for dementia to establish concretely. Signs of dementia include memory problems, confusion, distractibility, slowed thinking, decreased concentration, disorientation, lack of motivation and hallucinations [14]. However, before the dementia is diagnosed as a result of Parkinson's other possibilities including Vit-B12 deficiency, Alzheimer s and Lewy body should be excluded.

The other neuro-psychiatric consequences of PD include [14]

- $\quad$ Sleep problems including waking up in the middle of the night or suddenly falling asleep during daytime.

- Cognitive problems such as dementia, hallucinations, loss of intellectual thinking occur in the later stages of the disease.

- Mood disorders such as anxiety and depression. 
- Orthostatic hypotension.

- $\quad$ REM behavior disorder.

\section{Diagnostic Tools}

As part of an NIH sponsored workshop, provisional diagnostic criteria was established that defines a constellation of clinical features not shared by other psychotic syndromes [15].

\section{Differential diagnosis}

The other causes of psychosis that should be ruled out before diagnosing PD include [15].

- Delirium related to a variety of metabolic, toxic, or infectious causes, as well as acute drug intoxications.

- Progressive supranuclear palsy dementia, corticobasal ganglionic degeneration dementia, Lewy Body Dementia.

- $\quad$ Psychosis associated with comorbid psychiatric conditions occurred very early in the course of PD (first 3 months), did not fluctuate during the day and night, and was characterized by, prominent paranoia, fear and nonvisual hallucinations.

- Bipolar and unipolar depression with psychotic features are usually distinguished by mood congruent (e.g., delusions of guilt, worthlessness in the case of psychotic depression) psychotic symptoms in the context of prominent affective symptoms. PD psychosis patients typically have mood unrelated psychotic symptoms (e.g., seeing cats running on the bed).

- In schizophrenia and schizoaffective disorder, the age of onset of psychotic symptoms is in late adolescence and early adulthood whereas in PD psychosis the onset of symptoms is late after PD is developed i.e. in elderly.

- Other differentials include brief psychotic disorder, delusional disorder and substance induced psychosis.

- $\quad$ These criteria are not a measure of severity of psychosis in PD. However, the need for treatment of PD psychosis remains a clinical decision. The immediate and long-term value of these criteria depends upon their acceptance, use, and appropriate modification by the PD clinical research community [15].

\section{Neuropsychiatric Symptoms Based Diagnostic Assessment}

\section{Hallucinations, delusions and psychosis}

In $\mathrm{PD}$, hallucinatory experiences tend to be present intermittently, lasting seconds to minutes at a time. Their frequency can vary, but most studies register the presence of hallucinations when they occur at least one time per week. Often, they occur several times per day. The hallucinatory experiences in PD are not uniform. Simplistic classifications such as benign-malignant, simple-complex or minormajor should be avoided until a scaling method has been developed and validated. Previous reports have suggested that hallucinations tend to occur during times of low ambient stimulation, most typically in the evening or when the patient is alone in a quiet environment.

a) The Rush Behavioral interview primarily assesses the severity of hallucinations of all modalities focusing on frequency in the past month: severity score: $0=$ none, $1=$ less than once weekly, $2=$ once or twice weekly, 3 = at least three times weekly and does not ascertain detailed information about the type and nature of hallucinations. Severe hallucinations are defined as a score of 3 [16].

To this end we developed the UM-PDHQ to systematically study hallucinatory experiences in PD [17].

b) The UM-PDHQ is a 20-item clinician-administered questionnaire that is completed during a structured interview in an outpatient clinic setting. The 20 items were derived through consultations with PD patients, caregivers, and a panel of experts. The core group met on a monthly basis for a period of 6 months to produce a working questionnaire and subsequent revisions were made to improve ease of administration. Questions were divided into two groups; a quantitative group that consists of 6 questions (modality, frequency, duration, insight, emotional burden) and a qualitative group that consists of 14 questions. The first item is a gating question to assess the presence or absence of hallucinations. It is derived from modifications to item 3 of the UPDRS part I, and item 14 of the NMSQuest for PD. The quantitative section of the UM-PDHQ is currently being validated and is used only for descriptive purposes as part of this study. Other scales include Unified Parkinson's disease Rating Scale: UPDRS; Parkinson's Psychosis Rating Scale; non-motor symptom questionnaire (NMSQuest).

\section{Anxiety}

a) Hospital Anxiety and Depression Scale, b) Beck Anxiety Inventory, c) Zung Self-rating Anxiety scale, Spielberger State Trait Anxiety Inventory, and d) Hamilton Anxiety Rating Scale.

\section{Restless leg syndrome (RLS) and periodic limb movements in sleep (PLMS)}

It is characterized by repetitive dorsiflexion of the foot, extension of the great toe, and may be accompanied by flexion of the knee and hip cause disruptions of sleep) have an important impact on quality of sleep in PD patients. Several case reports demonstrate onset or worsening of RLS with use of antidepressants or antipsychotics like risperdone, aripiprazole, and quetiapine. The prevalence of PLMS in $\mathrm{PD}$ is approximately $60 \%$ and correlates with severity of PD motor features.The diagnosis is by Polysomnography [16].

\section{Delirium}

Cognitive dysfunction can be detected early in PD through neuropsychological testing; however, impairment of cognition is often insidious and may not be appreciated until symptoms become severe. other symptoms like Apathy, Impulse control disorders, Dopamine dysregulation syndrome, Punding can also be diagnosed earlier through neuropsychological testing [16].

\section{Excessive day time Sleepiness (EDS)}

Common complaint by PD patients is excessive daytime sleepiness, and verified by a Multiple Sleep Latency Test, which demonstrates a decreased latency of time to sleep with frequent periods of daytime sleep [16].

\section{Depression}

Depression occurs in approximately half of PD patients and is a significant cause of functional impairment for PD patients. It is postulated that depression is one of the four cardinal NMS that may predict development of PD others being olfaction, REM sleep disorder, depression and constipation. Formal testing through systematic questionnaires are diagnostically useful in the clinic, and serial testing can reveal changes over time to guide more effective treatment. Valid assessment tools to evaluate depression in PD include: 


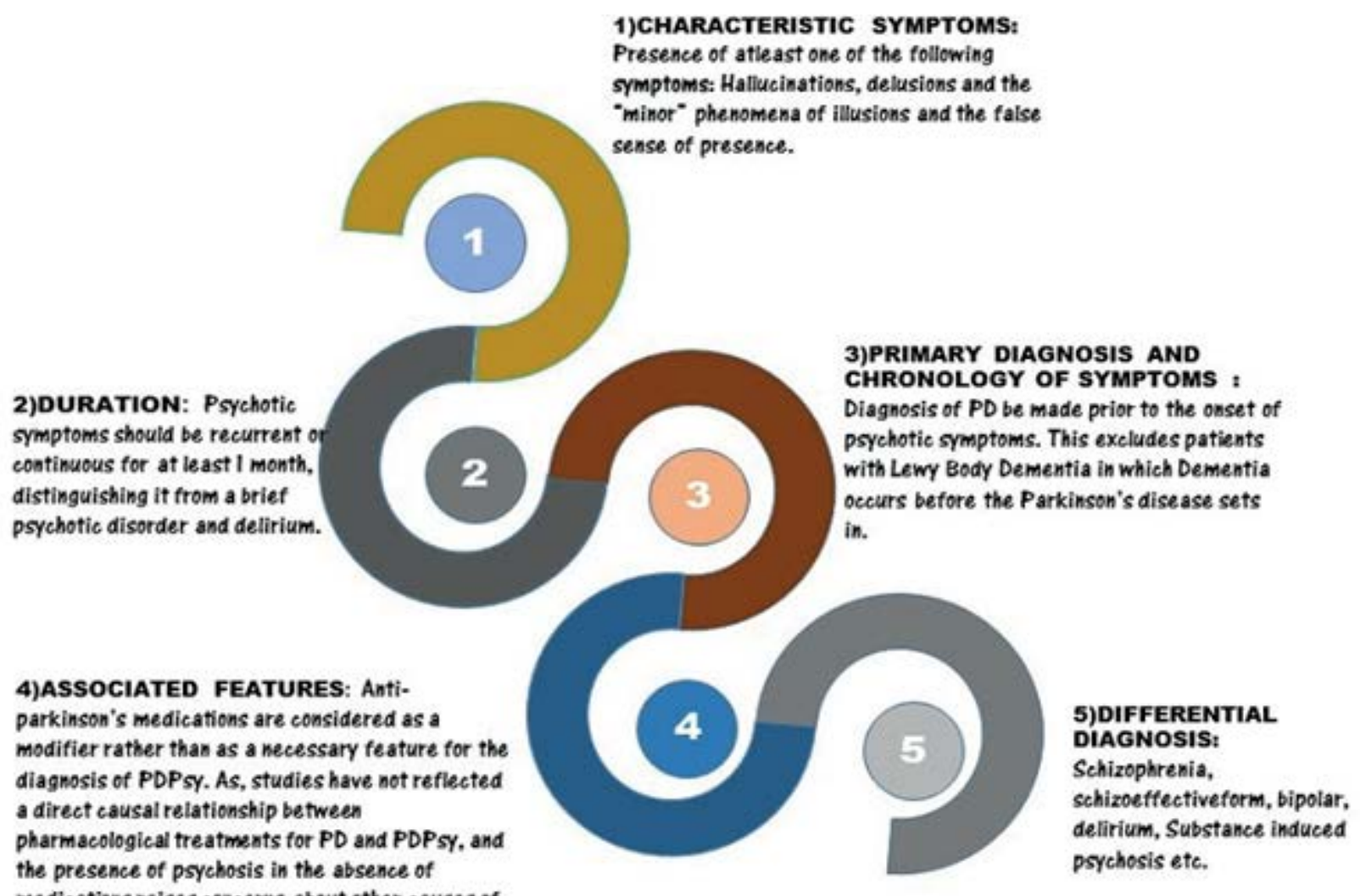

medications raises coscerns about other causes of

psychosis.

Figure 1: Diagnostic criteria aof Parkinson's disease Psychosis.

a) Beck Depression Inventory (21 question multiple choice selfreport inventory, one of the most widely used psychometric tests for measuring the severity of depression).

b) Hamilton Depression rating scale and Montgomery-Asberg Depression Rating Scale

c) Geriatric DRS

d) Hospital Anxiety and Depression scale

\section{Rapid eye movement Behavioral Disorder (RBD)}

It is seen in $25-50 \%$ of PD. Polysomnography is the most important diagnostic tool and demonstrates increased chin tone and limb movements during REM sleep in RBD. Diagnosis can also be made clinically with patient and bed partner reports, though sensitivity is only approximately $30 \%$.

\section{Sleep Disordered Breathing (SDB)}

Consists of either a deficit in the drive to breathe as in central sleep apnea, or may be due to an blockage of the airway as seen in obstructive sleep apnea (OSA). Polysomnography confirms the diagnosis and can direct treatment, which frequently includes application of CPAP devices during sleep [16].

The Psychotic symptoms compromise the quality of life more intensively compared to the motor symptoms, but still this spectrum of PD is under recognized. Despite the high prevalence and impact on disease burden, they often go undiagnosed due to factors including a lack of awareness and reporting by patients or insufficient interrogation by their physicians. Thus demanding the use of assessment tools for early diagnosis and prompt management.

\section{Imaging techniques}

Functional neuroimaging studies using fMRI showed increased activation of visual association cortex, decreased activation of primary visual cortex and increased activity in the frontal cortex in patient who experiences visual hallucinations [17,18]. Another studies showed that impairment of the ventral and lateral extra striate visual cortex in Parkinson's disease patients indicates a risk for the occurrence of VHs [19].

\section{Management}

While treating psychosis in PD, we should also check the medications of the victim as they may act as an important risk factor for the development psychotic symptoms [20]. Along with dopaminergic drugs, many other drugs such as hypnotics, narcotics, anxiolytics, and antidepressants can also contribute to the expression of psychosis. Postoperative and hospital induced psychosis [21] occur in $<60 \%$ of patients with $\mathrm{PD}$ (2.8-8.1 times more frequent than the general geriatric 
population), and this usually improves when patients return home [22]. Treatment of the underlying conditions may be all that is needed to reverse the psychiatric symptoms in these cases.

Four approaches used in treating drug-induced psychosis in PD are

1. Reduce the anti-PD drugs, and this persists as standard practice;

2. "Drug holiday" in which all anti-PD drugs were discontinued for up to 2 weeks; [23-27]

3. Addition of a neuroleptic [28,29], preferably of low potency;

4. Use of electroconvulsive therapy (ECT) [30]

\section{Medication reduction}

The most effective strategy in the treatment of psychosis in PD is the reduction of anti-PD drugs. Patients who are on multiple antiPD medications, it is recommended to decrease the medications in the following sequence: anticholinergics, selegiline, amantadine, dopamine agonists, catechol-O-Methyltransferase (COMT) inhibitors and lastly levodopa [31]. The doctors should consider the use of shortacting levodopa rather than long-acting versions because short-acting levodopa has a lower risk for the accumulation of adverse side effects. If the decrease in anti-PD medicines to the lowest dose tolerable without the worsening of motor symptoms does not develop psychosis, then the addition of an antipsychotic agent should be considered.

\section{Drug holidays}

Drug holidays have demonstrated to improve psychosis, and some patients sustained a hallucination-free state for $<12$ months [25-27]. However, these holidays have their set of restrictions and are considered to be physically and psychologically debilitating [22,24,32]. The continuation of the medications that induce the problem are essential after the holiday but at lower doses, resulting in lessened control of motor dysfunction. Drug holiday should not be considered as part of a plan to manage psychosis in PD [33,34].

\section{Antipsychotic medications}

The atypical antipsychotic drugs are considered as the first line in the treatment of psychosis in PD as they have fewer side effects as compared with typical antipsychotics. The atypical antipsychotics produce fewer and less severe elevation of serum prolactin levels and extrapyramidal symptoms, presumably due to dual serotonindopamine antagonism [35,36].

There are six atypical antipsychotic drugs marketed in the US: clozapine, risperidone, olanzapine [37], quetiapine, ziprasidone, and aripiprazole. The differences among these drugs lie in their relative tendencies to worsen motor symptoms, and the physician's choice of an antipsychotic agent is based largely on its unique side effect profile. Given the adverse consequences associated with the use of neuroleptics, the duration of treatment is an important consideration.

Clozapine, a di-benzodiazepine derivative, is the only atypical antipsychotic approved for the treatment of psychosis in PD based on meta-analysis done in 2007 [38]. Three well-designed placebocontrolled studies demonstrate its efficacy and tolerability in PD patients. In the studies, low-dose clozapine was found to be significantly better than placebo in decreasing positive symptoms of psychosis. There was no deterioration in global cognition and Parkinsonism [39-41].

Three placebo- controlled studies of olanzapine showed significant worsening of Parkinsonism in all the three studies and with a higher dropout rate in one study $[42,43]$. There have been two placebocontrolled trials of quetiapine for psychosis in PD. In the first study, no significant difference between placebo and quetiapine (dosage up to $200 \mathrm{mg} /$ day) was reported for any efficacy measures and no worsening of Parkinsonism was found with its treatment [41]. In the second study, quetiapine (mean dosage $119 \mathrm{mg}$ /day) showed no efficacy for the treatment of PD psychosis, and the dropout rate was 45\% [42].

There have been several studies in which clozapine is compared with other antipsychotics in the treatment of PD psychosis. In a study, a comparison of risperidone versus clozapine demonstrated an improvement on the Brief Psychiatric Rating Scale (BPRS) psychosis cluster score in the risperidone group. But the difference between two groups was not significant; there was no change seen in Unified Parkinson's Disease Rating Scale motor score [43]. In a randomized, double-blind study between olanzapine and clozapine was stopped before time because of exacerbation of Parkinsonism in olanzapinetreated patients. Finally, the result of single-blind randomized comparator study of quetiapine versus clozapine showed a significant increase in total BPRS score with medication, with no significant difference between both the groups. Both the groups do not show worsening in Unified Parkinson's Disease Rating Scale Motor Score. According to the literature on antipsychotics in PD, only clozapine can be fully recommended for the treatment of drug-induced psychosis in PD [38].

Aripiprazole, according to its mechanism of action is a partial agonist at D2 receptors. Due to its mechanism of action it was thought to be a promising drug for treating psychosis in PD patients, but two studies conducted at varying doses reported negative findings with a significant worsening of Parkinsonism or lack of improvement in psychosis leading to discontinuing of treatment by the patients $[44,45]$. In a case series, ziprasidone was used at a dose (20-40 $\mathrm{mg}$ /day) for the treatment of PD psychosis, $83 \%$ patients completed 12 weeks of treatment, with a notable overall improvement in total Neuropsychiatric Inventory score and no variation in the Unified Parkinson's Disease Rating Scale motor score [46].

\section{New medications}

But recently, non-dopaminergic, selective 5-HT2A inverse agonist/ antagonist, pimavanserin, has shown to have an antipsychotic like activity in an animal model of PD. Also pimavanserin was proved safe, effective and well-tolerated with high antipsychotic activity and lacks side effects such as sedation or motor impairment, which is usually seen with other available antipsychotics. Therefore, pimavanserin is considered superior to the available antipsychotics such as clozapine and quetiapine [47].

The safety of all antipsychotics in PD still needs to be better demonstrated and the alternatives for antipsychotics in the treatment of psychosis in PD need to be found as well. There is a little evidence that cholinesterase inhibitors and memantine may have both some benefits in the PD, including ameliorating the psychotic symptoms, but still further studies are needed to deal with these issues [48].

\section{Electroconvulsive therapy (ECT)}

ECT has shown its effectiveness in treating psychiatric disorders such as schizophrenia, medication-resistant depression and was found to improve motor function as well $[46,49-53]$ even in patients not having depression with PD [54]. However, the use of ECT in treating psychosis is limited to case reports; but it seems like ECT may be 
useful in decreasing psychotic symptoms in PD especially when there is concurrent depression and/or pharmacologic treatments have been unsuccessful. Most of the patients experienced a reduction of psychotic symptoms after administration of ECT [30,54,55]. However, Hurwitz et al. [55] indicated that efficacy persisted for 5 and 6 months in their two patients, suggested that Factor et al., [30] psychosis was controlled transiently. They also suggested that long-term medical therapy with clozapine was needed to maintain a psychosis-free state. As we know for the administration of ECT patients may require hospitalization and its side effect include confusion and cognitive deficits. So the use of ECT should only be considered when pharmacological treatments are unsuccessful.

\section{Prevention of Neuro Psychiatric Issues in PD}

\section{Early detection of pre-disposing factor}

In order to prevent the occurrence of psychosis in PD patients, we should also be familiar with some of the other factors contributing to psychosis. Beyond the dopaminergic treatments as the cause of PD psychosis, some other studies have shown that depression, cognitive impairment, motor severity, axial features, visual dysfunction and sleep-wake cycle disturbances may contribute to it. In the geriatric population, pulmonary and urinary infections, cerebral hypo perfusion states, endocrine disorders and even social stress may precipitate psychosis in PD [4]. Starting with the different sources that lead to PD psychosis, we cannot rule out autonomic dysfunctions, that has a high incidence in $\mathrm{PD}$, especially involving the bladder which predisposes to UTI's, one of the studies concluded that the neurology might be the better specialty handling PD cases, as primary care physicians may not be very well familiar with the subacute UTIs, leading to worsening of psychosis, confusion and motor symptoms (which may be commonly mistaken for disease progression).

Also, the non-motor symptoms of PD are frequently overlooked, either due to limited time spent with the patients, insights of the patient and caregiver that their psychosis is not related to the disease, or due to ignorance of the physician who may focus only towards the motor symptoms or his expectation that non-motor symptoms may be managed by family doctor. They do not pay much attention to the symptoms like anxiety, fatigue, depression or insomnia. Even though the neurologist might not be the best person to treat such kind of symptomatology, but at least they are able to identify them and refer the PD patient to the right specialty in order to prevent the further progression of non-motor symptoms.

\section{Drugs}

- $\quad$ There is not enough evidence for the treatment of non-motor symptoms with dopaminergic drugs. The dopamine agonists, such as pramipexole, and MAO inhibitors, such as selegiline, have insufficient evidence to prove as an anti-depressant. Even though dopamine agonists have been proved to be effective in restless leg syndrome, no clinical trials address its effectiveness in restless leg syndrome in PD patients. Rivastigmine has been proved efficacious in dementia associated with PD in one of the published studies [56].

- The prevention of psychosis in PD requires a multidimensional approach, there is a necessity for in-depth work up for metabolic/infectious disorders. The patient's living conditions needs to be thoroughly assessed to determine if hospitalization is required.

- Unnecessary psychoactive medications should be discontinued, including the less potent dopaminergic drugs [57]. Many non-motor symptoms such as constipation, dysautonomia, cognitive and olfactory deficits do not generally respond to dopaminergic treatments, in fact there are certain conditions such as orthostatic hypotension and dopamine dysregulation syndrome, that are indeed precipitated by dopamine agonists. Anti-parkinsonian drugs have also been associated with excessive daytime sleepiness, resulting in road traffic accidents due to its sedative effects [56]. These dopaminergic agents also affect REM sleep. Sleep hygiene should be the first line of treatment. Modafinil was not as efficacious as placebo in excessive daytime sleepiness. There is also not enough efficacy evidence for dopaminergic agents like melatonin, pergolide, etc. But a long-active dopamine agonist, cabergoline, has been considered effective [58].

- $\quad$ Also high dose levodopa has been associated with occasional anxiety and mania episodes [56] SSRI's are the first line of treatment for anxiety episodes, with other options being TCA's and BZ's. But BZ's has been associated with increased risk of falls in geriatric population, and should be used for minimum period of time and at the lowest possible dose

- The possibility of cholinergic neuronal degeneration may also be associated with psychosis in PD. Donepezil, an acetylcholinesterase inhibitor, has been shown to prevent psychosis in PD patients.

- As psychosis is generally overlooked, it is better to assess PD patients using a Parkinson Psychosis Questionnaire (PPQ) every 4 weeks, which contains 4 categories: sleep disturbance, hallucinations, delusions, and orientations [59].

- Some of the non-pharmacologic treatment includes psychotherapy, relaxation techniques, sleep hygiene and social measures for adaptation to PD. CBT, rTMS and DBS have also been proved efficacious in the management of anxiety symptoms associated with depression in PD patients [58].

\section{Management of depression}

Depression and PD both follow a malicious cycle. Presence of either one increases the risk of other. There are a few steps to be followed in the management of depression. First of all, we should review all the medications, and assess the relationship of medications with depression, and if needed, dosage should be adjusted. If it does not help, we should look carefully into other causes and start treating for depression. In mild cases, psychotherapy and CBT should be used as first line treatment. In moderate cases, one of the antidepressants and CBT should be used and in severe cases, ECT could be an option. TCA's are said to be effective and devoid of anticholinergic side effects [58].

\section{Conclusion}

The neuropsychiatric component of PD has a greater impact on quality of life and healthcare costs than the cardinal motor symptoms that define the disease. Many of these symptoms are not recognized by the practitioners and are often not recognized by PD patients or the caregivers. Therefore, more improved quality scales and integrated methods for the assessment of non-motor symptoms are needed in order to timely recognize the symptoms, which will in turn reduce the cost of care of patients associated with its poor recognition. Neuropsychiatry in PD is challenging and can often be compromised by the importance of addressing the co-existing motor symptoms, thereby complicating the balance needed to alleviate the psychiatric manifestations while maintaining sufficient control of motor symptoms. Various diagnostic tools and preventive strategies as discussed above are now available including a variety of pharmacologic and non-pharmacologic means. Nevertheless, the early recognition of these symptoms is important so as to not lose an opportunity to make a positive and potentially 
significant impact on the Parkinson patient's quality of life.

\section{References}

1. Inzelberg R, Kipervasser S, Korczyn AD (1998) Auditory hallucinations in parkinson's disease. Journal of Neurology, Neurosurgery \& Psychiatry 64(4): 533-5.

2. Zahodne LB, Fernandez HH (2008) Pathophysiology and treatment of psychosis in parkinson's disease. Drugs \& aging 25(8): 665-82.

3. Fénelon G, Alves G (2010) Epidemiology of psychosis in parkinson's disease. Journal of the neurological sciences 289(1): 12-7.

4. Rabey JM (2009) Hallucinations and psychosis in parkinson's disease. Parkinsonism \& related disorders. 15: S105-S10.

5. Forsaa EB, Larsen JP, Wentzel-Larsen T, Goetz CG, Stebbins GT, et al. (2010) A 12-year population-based study of psychosis in parkinson disease. Archives of Neurology 67(8): 996-1001.

6. Stacy M, Jakel R (2014) Parkinson's disease psychosis. Journal of Parkinsonism and Restless Legs Syndrome. 4: 41-51.

7. Wolters EC (2001) Intrinsic and extrinsic psychosis in parkinson's disease. Journal of Neurology. 248(3): 22-7.

8. Zoldan J, Friedberg G, Goldberg-Stern H, Melamed E (1993) Ondansetron for hallucinosis in advanced parkinson's disease. The Lancet. 341(8844): 562-3.

9. Whitehouse PJ (1987) Clinical and neurochemical consequences of neuronal loss in the nucleus basalis of meynert in parkinson's disease and alzheimer's disease. Advances in neurology 45: 393.

10. Perry E, Walker M, Grace J, Perry R (1999) Acetylcholine in mind: A neurotransmitter correlate of consciousness? Trends in neurosciences 22 : 273-80.

11. Wint DP, Okun MS, Fernandez HH (2004) Psychosis in parkinson's disease Journal of Geriatric psychiatry and Neurology 17(3): 127-36.

12. Thanvi B, Lo T, Harsh D (2005) Psychosis in parkinson's disease. Postgraduate medical journal. 81(960): 644-6.

13. Christopher G Goetz (2003) Movement Disorder Society Task Force on Rating Scales for Parkinson's Disease. The unified parkinson's disease rating scale (updrs): Status and recommendations. Movement disorders: official journal of the Movement Disorder Society 18(7): 738.

14. Farlow J, Pankratz ND, Wojcieszek J, Foroud T (2014) Parkinson disease overview.

15. Ravina B, Marder K, Fernandez HH, Friedman JH, McDonald W, et al. (2007) Diagnostic criteria for psychosis in parkinson's disease: Report of an ninds, nimh work group. Movement Disorders. 22(8): 1061-8.

16. Van Laar AD, Jain S (2004) Non-motor symptoms of parkinson disease: Update on the diagnosis and treatment. The neurologist.10(4): 185.

17. Papapetropoulos S, Katzen H, Schrag A, Singer C, Scanlon BK, et al. (2008) A questionnaire-based (um-pdhq) study of hallucinations in parkinson's disease. BMC neurology 8(1): 21.

18. Stebbins GT, Goetz CG, Carrillo MC, Bangen KJ, Turner DA, et al. (2004) Altered cortical visual processing in pd with hallucinations: An fmri study. Neurology. 63(8): 1409-16.

19. Meppelink AM, De Jong BM, Renken R, Leenders KL, Cornelissen FW, et al. (2009) Impaired visual processing preceding image recognition in parkinson's disease patients with visual hallucinations. Brain. 132(Pt 11): 2980-93.

20. Henderson MJ, Mellers JD (2000) Psychosis in parkinson's disease: 'Between a rock and a hard place'. International Review of Psychiatry 12(4): 319-34.

21. Golden WE, Lavender RC, Metzer WS (1989) Acute postoperative confusion and hallucinations in parkinson disease. Annals of internal medicine 111(3): 218-22.

22. Factor SA, Molho ES, Podskalny GD, Brown D (1994) Parkinson's disease: Drug-induced psychiatric states. Advances in neurology 65: 115-38.

23. Feldman RG, Kaye JA, Lannon MC (1986) Parkinson's disease: Follow $\square$ up after "drug holiday". The Journal of Clinical Pharmacology 26(8): 662-7.

24. Friedman JH (1985) 'Drug holidays' in the treatment of parkinson's disease: A brief review. Archives of internal medicine. 145(5): 913-5.
25. Klawans H, Goetz C, Tanner C, Nausieda P, Weiner W (1983) Levodopa-free periods (" drug holidays") in the management of parkinsonism. Advances in neurology. 37: 33.

26. Sweet RD, Lee JE, Spiegel HE, McDowell F (1972) Enhanced response to low doses of levodopa after withdrawal from chronic treatment. Neurology. 22(5): 520

27. Weiner WJ, Koller WC, Perlik S, Nausieda PA, Klawans HL (1980) Drug holiday and management of parkinson disease. Neurology 30(12): 1257-61.

28. Crow T, Johnstone EC, McClelland H (1976) The coincidence of schizophrenia and parkinsonism: Some neurochemical implications. Psychological medicine 6(02): 227-33.

29. Hale MS, Bellizzi J (1980) Single case study: Low dose perphenazine and levodopa/carbidopa therapy in a patient with parkinsonism and a psychotic illness. The Journal of nervous and mental disease. 168(5): 312-4.

30. Factor SA, Molho ES, Brown DL (1995) Combined clozapine and electroconvulsive therapy for the treatment of drug-induced psychosis in parkinson's disease. The Journal of neuropsychiatry and clinical neurosciences 7(3): 304-7.

31. Fernandez HH, Friedman JH (1999) The role of atypical antipsychotics in the treatment of movement disorders. CNS drugs 11(6): 467-83.

32. Factor S, Singer C (1992) Neuroleptic malignant syndrome. Drug induced movement disorders. 199-230.

33. Keltner NL, Johnson V (2102) Aripiprazole: A third generation of antipsychotics begins? Perspectives in psychiatric care 38(4): 157-9.

34. Noel JM (2007) Ashp therapeutic position statement on the use of secondgeneration antipsychotic medications in the treatment of adults with psychotic disorders. American journal of health-system pharmacy 64(8): 863-76.

35. Mayeux R, Stern Y, Mulvey K, Cote L (1985) Reappraisal of temporary levodopa withdrawal (drug holiday) in parkinson's disease. New England Journal of Medicine 313(12): 724-8.

36. Friedman JH, Fernandez HH (2000) The nonmotor problems of parkinson's disease. The Neurologist 6(1): 18-27.

37. Breier A, Sutton VK, Feldman PD, Kadam DL, Ferchland I, et al. (2102) Olanzapine in the treatment of dopamimetic-induced psychosis in patients with parkinson's disease. Biological psychiatry 52(5): 438-45.

38. Frieling H, Hillemacher T, Ziegenbein M, Neundörfer B, Bleich S (2007) Treating dopamimetic psychosis in parkinson's disease: Structured review and metaanalysis. European neuropsychopharmacology 17(3): 165-71.

39. Friedman J, Lannon M, Comella C, Factor S, Kurlan R, et al. (1999) Low-dose clozapine for the treatment of drug-induced psychosis in parkinson's disease. New England Journal of Medicine. 340(10): 757-63.

40. French Clozapine Parkinson Study Group. Clozapine in drug-induced psychosis in parkinson's disease. The Lancet. 1999, 353(9169): 2041-2.

41. Ondo WG, Tintner R, Dat Voung K, Lai D, Ringholz G (2005) Double blind, placebo $\square$ controlled, unforced titration parallel trial of quetiapine for dopaminergic $\square$ induced hallucinations in parkinson's disease. Movement Disorders. 20(8): 958-63.

42. Ondo WG, Levy JK, Vuong KD, Hunter C, Jankovic J (2102) Olanzapine treatment for dopaminergic $\square$ induced hallucinations. Movement Disorders. 17(5): 1031-5.

43. Rabey JM, Prokhorov T, Miniovitz A, Dobronevsky E, Klein C (2007) Effect of quetiapine in psychotic parkinson's disease patients: A double $\square$ blind labeled study of 3 months' duration. Movement disorders 22(3): 313-8.

44. Fernandez HH, Trieschmann ME, Friedman JH (2004) Aripiprazole for drug induced psychosis in parkinson disease: Preliminary experience. Clinical neuropharmacology 27(1): 4-5.

45. Friedman JH, Berman RM, Goetz CG, Factor SA, Ondo WG et al. (2006) Open $\square$ label flexible $\square$ dose pilot study to evaluate the safety and tolerability of aripiprazole in patients with psychosis associated with parkinson's disease. Movement disorders 21(12): 2078-81.

46. Gómez-Esteban JC, Zarranz JJ, Velasco F, Lezcano $E$, Lachen M, et al. (2005) Use of ziprasidone in parkinsonian patients with psychosis. Clinical neuropharmacology 28(3): 111-4.

47. Hubbard D, Hacksell U, McFarland K (2013) Behavioral effects of clozapine, 
pimavanserin, and quetiapine in rodent models of parkinson's disease and parkinson's disease psychosis: Evaluation of therapeutic ratios. Behavioural pharmacology 24(7): 628-32.

48. Herrmann N, Marras C, Fischer HD, Wang X, Anderson GM, et al. (2013) Management of neuropsychiatric symptoms in long-term care residents with parkinson's disease. Drugs \& aging 30(1): 19-22.

49. Abrams R (1989) Ect for parkinson's disease. The American journal of psychiatry 146(11): 1391

50. Douyon R, Serby M, Kluthcko B, Rotrosen J (1989) Ect and parkinson's disease revisted: A" naturalistic" study. The American journal of psychiatry. 146(11): 1451-1455.

51. Ellis T, Cudkowicz ME, Sexton PM, Growdon JH (2014) Clozapine and risperidone treatment of psychosis in parkinson's disease. The Journal of neuropsychiatry and clinical neurosciences.

52. Fochtmann $L$ (1988) A mechanism for the efficacy of ect in parkinson's disease. The Journal of ECT. 4(4): 321-7.

53. Stern MB (1991) Electroconvulsive therapy in untreated parkinson's disease.
Movement Disorders 6(3): 265.

54. Balldin J, Eden S, Granerus A-K, Modigh K, Svanborg A, et al. (1980) Electroconvulsive therapy in parkinson's syndrome with "on-off" phenomenon. Journal of Neural Transmission 47(1): 11-21.

55. Hurwitz T, Calne D, Waterman K (1988) Treatment of dopaminomimetic psychosis in parkinson's disease with electroconvulsive therapy. The Canadian journal of neurological sciences Le journal canadien des sciences neurologiques. 15(1): 32-4.

56. Chaudhuri KR, Healy DG, Schapira AH (2006) Non-motor symptoms of parkinson's disease: Diagnosis and management. The Lancet Neurology 5(3): 235-45.

57. Robottom BJ, Weiner WJ, Factor SA (2011) Movement disorders emergencies part 1: Hypokinetic disorders. Archives of neurology 68(5): 567-72.

58. Grover S, Somaiya M, Kumar S, Avasthi A (2015) Psychiatric aspects of parkinson's disease. Journal of neurosciences in rural practice 6(1): 65-76.

59. Sawada H, Oeda T (2013) Protocol for a randomised controlled trial: Efficacy of donepezil against psychosis in parkinson's disease (edap) BMJ open 3(9): e003533.
Citation: Farheen SA, Shah P, Gill M, Ayaz S, Singh S, et al. (2015) Translationa Science of Psychosis in Parkinson's Disease. Clin Depress 1: 102.

\section{OMICS International: Publication Benefits \& Features}

\section{Unique features:}

Increased global visibility of articles through worldwide distribution and indexing

Showcasing recent research output in a timely and updated manner

- Special issues on the current trends of scientific research

Special features:

700 Open Access Journals

50,000 editorial team

Rapid review proces

Quality and quick editorial, review and publication processing

- Indexing at PubMed (partial), Scopus, EBSCO, Index Copernicus and Google Scholar etc

Sharing Option: Social Networking Enabled

- Authors, Reviewers and Editors rewarded with online Scientific Credits

- Better discount for your subsequent articles

Submit your manuscript at: www.omicsonline.org/submission/ 